\title{
Tension wood provides insight into structural changes in biomass resulting from chemical pretreatment
}

\author{
Volker Urban ${ }^{1}$ (urbanvs@ornl.gov), Sai Venkatesh Pingali, ${ }^{1}$ Daisuke Sawada, ${ }^{1}$ Udaya Kalluri, ${ }^{1}$ Hugh
} M. O’Neill, ${ }^{1}$ Paul Langan, ${ }^{1}$ and Brian H. Davison ${ }^{1}$

${ }^{1}$ Oak Ridge National Laboratory, Oak Ridge, Tennessee

Plant cell walls comprise the bulk of lignocellulosic biomass. Therefore, a greater understanding of the chemistry, architecture, physical and mechanical properties of cell walls is essential to improve biomass-based biofuel production. The work presented here furthers understanding of biomass properties underlying recalcitrance to enzyme-based sugar (ethanol substrate) release. The spatiotemporal progression in chemical and anatomical changes that occur in growing plants under tension stress is investigated. An earlier neutron diffraction study of hydrogenated and partially deuterated poplar tension wood found that, unlike fibers from the ramie plant, the characteristic diffraction peaks were not present. The authors postulated that the contribution of laterally aligned microfibrils complicated the signal from the majority of the microfibrils, which are longitudinally aligned, but did not further investigate the tension wood structure. Here, we report structural studies of tension wood using small-angle neutron scattering at the Bio-SANS instrument. ${ }^{1}$ We studied a similar type of system namely tension wood in which external force such as a bending stress causes overexpression of cellulose in the stressed side (tension). We used intact microtomed samples of normal and tension wood that resulted in anisotropic scattering. A crystalline core $\left(R_{\mathrm{g}}=10 \sim 11 \AA\right)$ is observed for normal wood. ${ }^{2}$ Meanwhile, the tension side showed evidence of different levels of associations between elementary fibrils, forming different aggregate sizes. Most interestingly, these aggregates are multiples of the elementary fibril. This propensity to associate due to the application of mechanical stress (also called tension) produces identical effects on the plant nanostructure as observed during most thermochemical pretreatments, especially dilute acid pretreatment.

\section{Reference}

1. The development of cell was partially funded by the Oak Ridge National Laboratory's Center for Structural Molecular Biology (CSMB), which is supported by the Office of Biological and Environmental Research, using facilities supported by the U.S. Department of Energy, managed by UT-Battelle, LLC under Contract No. DE-AC05-00OR22725.

2. S.V. Pingali et al., Biomacromolecules 2010, 11, 2329-2335.

Oak Ridge National Laboratory is managed by UT-Battelle, LLC for the U.S. Department of Energy under contract no. DE-AC05-00OR22725. This program is supported by the Office of Biological and Environmental Research in the DOE Office of Science. This research used resources at the High Flux Isotope Reactor, a DOE Office of Science User Facility operated by the Oak Ridge National Laboratory. 